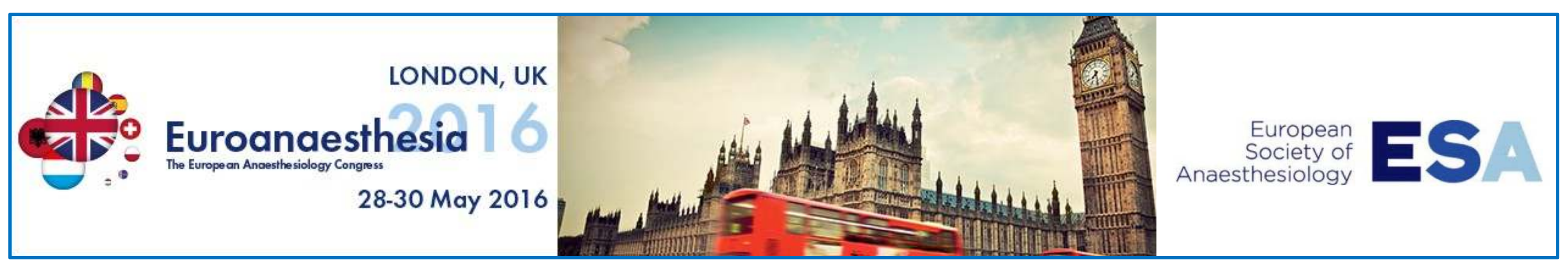

\title{
Perioperative hyperoxia and long term mortality under colorectal surgery
}

Castelltort Mascó L., Sadurní Sardà M., García Bernedo C., Bosch Duran L., Gallart Gallego LI., Escolano Villen F.

Parc de Salut Mar, Dept of Anaesthesiology. Institut Hospital del Mar d'Investigacions Mèdiques (IMIM). Universitat Autònoma de Barcelona (UAB).

Barcelona, Spain.

Background and Goal of Study: Perioperative hyperoxia $\left(\mathrm{FiO}_{2}>80 \%\right)$ appears to reduce the incidence of surgical site infection in colorectal surgery (CRS) (I). Despite of this benefit, Meyhoff et al. observed an increased long-term mortality in patients receiving hyperoxia during CRS (2). This is a controversial topic, and the goal of this study was to assess long term mortality in our patients submitted to CRS according to the $\mathrm{FiO}_{2}$ received, hyperoxia $\left(\mathrm{FiO}_{2} 80 \%\right)$ or standard $\left(\mathrm{FiO}_{2}<50 \%\right)$.

Materials and Methods: A retrospective cohort study to assess survival was performed with telephone survey in patients who underwent elective colorectal surgery from October 201I to December 2013. Follow up date to assess survival status was January 2015.

Demographic and surgical variables and cancer staging were analyzed. The main outcome was the mortality in follow up period. Survival in two groups, hyperoxia vs standard, was analyzed using Kaplan-Meier statistics.

Results and Discussion: A total of 223 patients were operated in the analyzed period, 129 receiving $<50 \%$ perioperative oxygen and 94 receiving $80 \%$. Mean duration of follow-up from surgery was $2.2(\mathrm{I} . \mathrm{I}-3.3) \mathrm{yr}$. No significant differences between groups were observed for cancer staging, ASA status, demographic and surgical variables (table I). Mortality rate at the end of follow-up was $14 \%$ in hyperoxia group versus $19 \%$ in group control $(p=0.6)$. Kaplan-Meier survival curve did not show significant differences between both groups (image I).

Conclusions: In our patients scheduled for elective CRS, mortality was similar in spite of the $\mathrm{FiO}_{2}$ received. These findings suggest that hyperoxia could be used until more evidence is available.

\section{References:}

I. Hovaguimian F et al. Anesthesiology 2013;1 19:303-16

2. Meyhoff CS et al. Anesth Analg. 20I2; I I5(4):849-54.

\begin{tabular}{|c|c|c|}
\hline & $\begin{array}{l}80 \% \text { oxygen } \\
\text { group (n 94) }\end{array}$ & $<50 \% \underset{\text { oxygen group }}{<50)}$ \\
\hline Sex (male:female) (\%) & $37.5 / 62.5$ & $26 / 74$ \\
\hline Age $(y)$ & $70 \pm 11$ & $68 \pm 13$ \\
\hline Body mass index $(\mathrm{kg} / \mathrm{m} 2)$ & $26 \pm 4.5$ & $26 \pm 3.9$ \\
\hline $\begin{array}{l}\text { ASA physical status } \\
\text { class (l:II:III) (\%) }\end{array}$ & $8 / 59 / 33$ & $6 / 64 / 30$ \\
\hline $\begin{array}{l}\text { Surgical procedure (\%) } \\
\text { Hemicolectomy } \\
\text { Sigmoid anterior section } \\
\text { Rectal anterior resection } \\
\text { Total or subtotal colectomy } \\
\text { Rectum resection with } \\
\text { abdominal-perineal repair }\end{array}$ & $\begin{array}{c}38 \\
34 \\
20 \\
6 \\
2\end{array}$ & $\begin{array}{c}33 \\
35 \\
22 \\
5 \\
5\end{array}$ \\
\hline $\begin{array}{l}\text { Stage of tumor } *(\%) \\
<=2 \\
>\quad 2\end{array}$ & $\begin{array}{l}58 \\
42\end{array}$ & $\begin{array}{l}60 \\
40\end{array}$ \\
\hline $\begin{array}{l}\text { Surgical approach } \\
\text { (\% laparoscopy/laparotomy) }\end{array}$ & $34 / 66$ & $44 / 56$ \\
\hline $\begin{array}{l}\text { Duration of surgery } \\
\text { (min) }\end{array}$ & $188 \pm 59$ & $195 \pm 70$ \\
\hline $\begin{array}{l}\text { Peroperative blood } \\
\text { transfusion (\%) }\end{array}$ & 21 & 22 \\
\hline
\end{tabular}

Values are median (5\%-95\% range). * AJCC/UICC TNM staging system for Colorectal Cancer (2010).

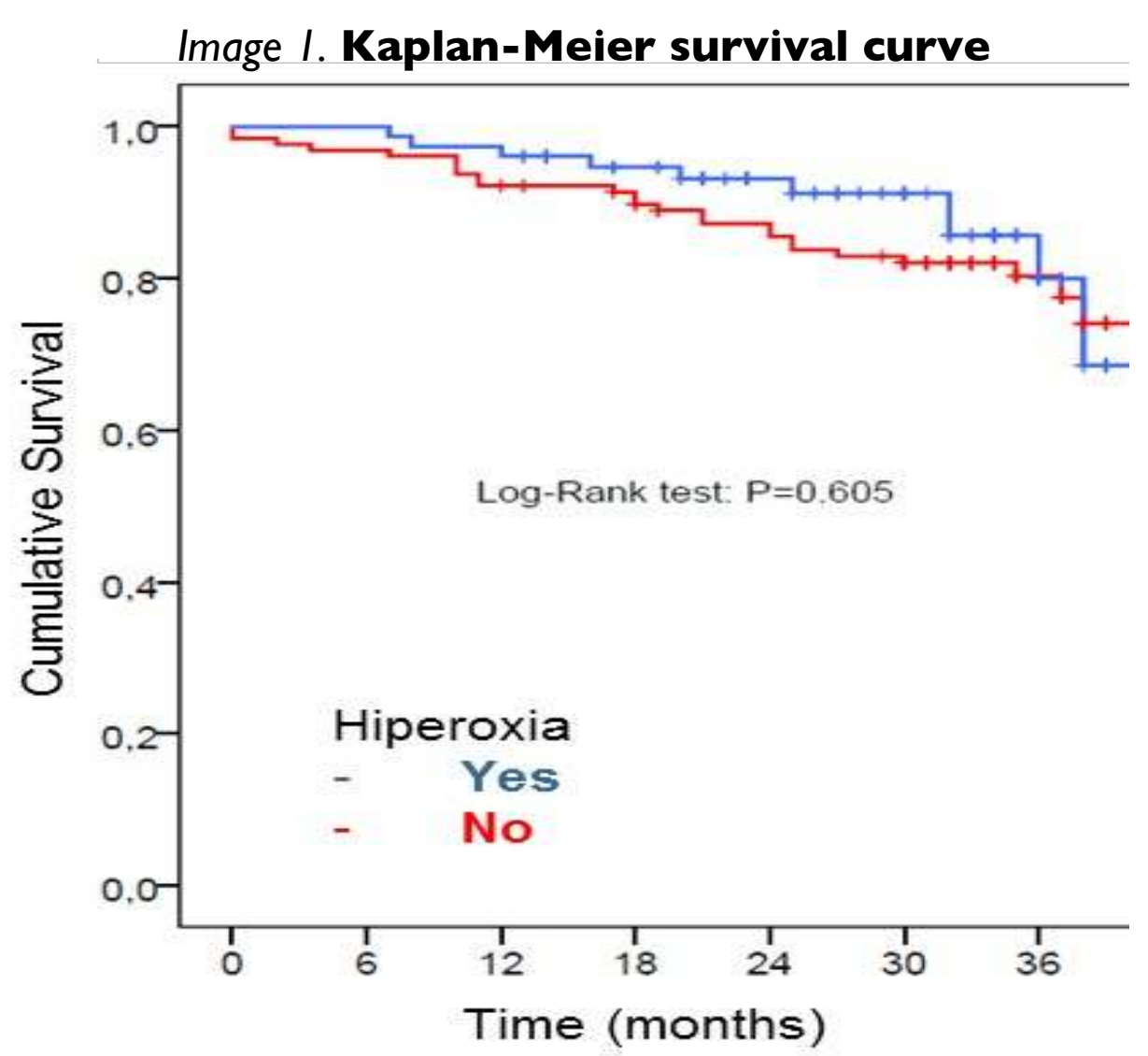

